\title{
STRONG SOLUTIONS AND INVISCID LIMIT FOR BOUSSINESQ SYSTEM WITH PARTIAL VISCOSITY*
}

\author{
LUCAS C.F. FERREIRA $^{\dagger}$ AND ELDER J. VILLAMIZAR-ROA ${ }^{\ddagger}$
}

\begin{abstract}
We consider the convection problem of a fluid with viscosity depending on temperature in either a bounded or an exterior domain $\Omega \subset \mathbb{R}^{N}, N=2,3$. It is assumed that the temperature is transported without thermal conductance (dissipation) by the velocity field which is described by the Navier-Stokes flow. This model is commonly called the Boussinesq system with partial viscosity. In this paper we prove the existence and uniqueness of strong solutions for the Boussinesq system with partial viscosity with initial data in $W^{2-\frac{2}{p}, p}(\Omega) \times W^{1, q}(\Omega)$. For a bounded domain $\Omega$, we also analyze the inviscid limit problem when the conductivity in the fully viscous Boussinesq system goes to zero.
\end{abstract}

Key words. Boussinesq system, partial viscosity, strong solutions, inviscid limit.

AMS subject classifications. 35Q35, 76B03, 76D09.

\section{Introduction}

The Boussinesq equations of hydrodynamics describe the evolution of the velocity $u$ of an incompressible fluid caused by forces depending on variations of the temperature $\theta$, which is transported with or without diffusion. When the kinematic viscosity $\nu$ and heat conductivity coefficient $\kappa$ are strictly positive constants, the Boussinesq system is given by the coupled PDEs

$$
\left\{\begin{array}{r}
\partial_{t} u-\nu \Delta u+u \cdot \nabla u+\nabla \pi=\theta f \text { in } Q_{T}, \\
\operatorname{div} u=0 \text { in } Q_{T}, \\
\partial_{t} \theta-\kappa \Delta \theta+u \cdot \nabla \theta=0 \text { in } Q_{T},
\end{array}\right.
$$

where $Q_{T} \equiv \Omega \times(0, T)$. Here $\Omega \subseteq \mathbb{R}^{N}(N \geq 2)$ represents the domain in which the fluid is occurring and $(0, T)$ stands for a time existence interval. The function $\pi: Q_{T} \rightarrow \mathbb{R}$ denotes the pressure and $f: Q_{T} \rightarrow \mathbb{R}^{N}$ is a given external field, usually the gravitational force.

The system (1.1) has been widely analyzed from the mathematical viewpoint and many results on existence, regularity and asymptotic behavior have been proved (see for instance $[23,6,25,14,15,16,29,18,1,5]$ and references therein). In these references, the reader can find global well-posedness results in the $2 \mathrm{D}$ case, global existence of weak solutions and local existence of strong solutions in the 3D case, regularity results for weak solutions, existence of asymptotically self-similar solutions, and stability results in several singular frameworks.

In this paper we are interested in the study of Boussinesq systems under the following two considerations. The first case is when the temperature is transported with no dissipation by the velocity field, that is, $\kappa=0$ in (1.1). This situation is of great interest due to applicability in several geophysical phenomena as well as its close connection with fundamental models such as Euler and Navier- Stokes equations

\footnotetext{
*Received: September 14, 2011; accepted (in revised form): June 22, 2012. Communicated by Alexander Kiselev.

L.C.F. Ferreira was supported by FAPESP and CNPq, Brazil.

†Universidade Estadual de Campinas, Departamento de Matemática, Rua Sérgio Buarque de Holanda, 651, CEP 13083-859, Campinas-SP, Brazil (lcff@ime.unicamp.br).

$\ddagger$ Universidad Industrial de Santander, Escuela de Matemáticas, A.A. 678, Bucaramanga, Colombia (jvillami@uis.edu.co).
} 
(see e.g. $[28,26,24,3,10,20,7,11,19]$ ). The second consideration is when the kinematic viscosity $\nu$ depends on temperature. The variation of the viscosity with the temperature may be important to a better description of the flow. Indeed, it is known that the viscosity of a typical liquid decreases with temperature whereas that of a typical gas increases (see [17]). The corresponding Boussinesq model describing the above two considerations is given by

$$
\left\{\begin{array}{r}
\partial_{t} u-\operatorname{div}\left(\nu(\theta)\left(\nabla u+\nabla^{*} u\right)\right)+u \cdot \nabla u+\nabla \pi=\theta f \text { in } Q_{T}, \\
\operatorname{div} u=0 \text { in } Q_{T}, \\
\partial_{t} \theta+u \cdot \nabla \theta=0 \text { in } Q_{T}
\end{array}\right.
$$

where the viscosity $\nu$ is a function $\nu: \mathbb{R} \rightarrow(0, \infty)$ with $\nu(\xi) \geq \nu_{0}>0$ for all $\xi \in \mathbb{R}$, and $\nu_{0}$ is a constant. We also assume $\nu(x)=k_{2}+\tilde{\nu}(x)$ with $k_{2}$ a positive constant and $\tilde{\nu}$ satisfying the below condition (1.6), which is compatible with $\nu(\xi) \geq v_{0}$ (see Remark 1.1). The symbol $\nabla^{*}$ denotes the transpose of the operator $\nabla$. The system (1.2) is completed with the following initial and boundary conditions:

$$
\left\{\begin{array}{l}
\left.(u, \theta)\right|_{t=0}=\left(u_{0}, \theta_{0}\right) \text { in } \Omega \\
u=0 \text { on } \Gamma_{T}=\partial \Omega \times(0, T) .
\end{array}\right.
$$

In this paper we consider $N=2,3$ and $\Omega \subseteq \mathbb{R}^{N}$ being either a bounded or an exterior domain with boundary $\partial \Omega$ smooth enough. These two kind of domains correspond to important physical situations. In particular, the case of an exterior domain to a sphere of radius $R$ is relevant in order to study the convection of a viscous incompressible fluid in the exterior of a ball heated through its surface $|x|=R$ (see e.g. [14, 18]). In the case of bounded domains, it is common to take $f=-g_{0} \vec{e}_{N}$ where $g_{0}$ is the gravitational constant. Recently, several authors have studied the system (1.2) on the whole space $\mathbb{R}^{N}$ with $f=-g_{0} \vec{e}_{N}$, and interesting results have been obtained for instance in $[10,20,7,11,19]$. However, a more physical situation is to consider the origin of the gravitational force outside the fluid domain, which occurs when selfgravitation effects are neglected (see [13]). Corresponding to such condition, one has an exterior domain $\Omega$ and $f(x) \approx g_{0} \nabla(1 /|x|)$ for $|x|$ large. In Remark 1.1 we comment how to cover the case $f(x)=g_{0} \nabla(1 /|x|)$.

Among others, the work [13] obtained existence theory and long-time behavior of solutions for the full viscous Oberbeck-Boussinesq approximation in exterior domains with both kinematic viscosity $\nu>0$ and the heat conductivity coefficient $\kappa>0$ being constant (see the system (1.4) below). Indeed, as far as we know, only a few works are devoted to the study of the Boussinesq system with partial viscosity $(\kappa=0)$ in bounded or exterior domains. For instance, we have found in the literature the work [22] where the authors studied the existence of classical solutions for (1.2)-(1.3) with $v>0$ being a constant, $\Omega$ a bounded domain, and $H^{3}$-initial data (see also [8] for $v=0)$.

We focus on the question of finding unique local strong solutions for (1.2)-(1.3) with initial data $\left(u_{0}, \theta_{0}\right)$ belonging to $W^{2-\frac{2}{p}, p}(\Omega) \times W^{1, q}(\Omega)$ and $\Omega$ being either a bounded or an exterior domain. Our results are obtained by means of an iterative approach combined with recent results due to Abels [2] for general nonstationary Stokes systems in unbounded domains (see Lemma 2.3). In order to perform the iterative scheme, we need to obtain a stability result in the $W^{1, p}$-framework for an associated transport equation (see Lemma 2.2). Afterwards, we consider the inviscid limit of local strong solutions, when $0 \leq \kappa(\theta) \leq \tilde{\kappa}$ and $\tilde{\kappa} \rightarrow 0$, for the fully Boussinesq 
system with viscosity depending on temperature in a bounded domain $\Omega$, which reads as

$$
\left\{\begin{array}{r}
\partial_{t} u-\operatorname{div}\left(\nu(\theta)\left(\nabla u+\nabla^{*} u\right)\right)+u \cdot \nabla u+\nabla \pi=\theta f \text { in } Q_{T}, \\
\operatorname{div} u=0 \text { in } Q_{T}, \\
\partial_{t} \theta-\operatorname{div}(\kappa(\theta)(\nabla \theta))+u \cdot \nabla \theta=g \text { in } Q_{T}
\end{array}\right.
$$

The inviscid limit when $\kappa(\theta)$ is constant, that is $\kappa(\theta) \equiv \tilde{\kappa} \rightarrow 0$, was discussed in [7] in the framework of $H^{m}\left(\mathbb{R}^{2}\right) \times H^{m}\left(\mathbb{R}^{2}\right)$.

1.1. Main results. Before stating the main results, we introduce some function spaces. Throughout this paper we use the classical notations and results of Sobolev spaces $W^{k, p}(\Omega)$. The letter $c$ represents a positive constant that may change from line to line.

For $1 \leq p<\infty$, and $0<T<\infty$, let $\mathcal{E}_{p, T}^{2,1}$ be the Banach space of functions $u \in$ $L^{p}\left(0, T ; W^{2, p}(\Omega)\right)$ such that $\partial_{t} u \in L^{p}\left(Q_{T}\right):=L^{p}\left(0, T ; L^{p}(\Omega)\right)$ endowed with the norm:

$$
\|u\|_{\mathcal{E}_{p, T}^{2,1}}:=\left\|\partial_{t} u\right\|_{L^{p}\left(Q_{T}\right)}+\|u\|_{L^{p}\left(Q_{T}\right)}+\|\nabla u\|_{L^{p}\left(Q_{T}\right)}+\|\nabla(\nabla u)\|_{L^{p}\left(Q_{T}\right)} .
$$

It is known that the space $\mathcal{E}_{p, T}^{2,1} \subset C\left([0, T] ; W^{2-\frac{2}{p}, p}(\Omega)\right)$, with continuous embedding, where $W^{2-\frac{2}{p}, p}(\Omega):=\left(L^{p}(\Omega), W^{2, p}(\Omega)\right)_{1-\frac{1}{p}, p}$.

Our main result reads as follows:

TheOREm 1.1. Let $0<T<\infty, N<p \leq q<\infty, f \in L^{p}\left(Q_{T}\right), u_{0} \in W^{2-\frac{2}{p}, p}(\Omega),\left.u_{0}\right|_{\partial \Omega}=$ 0 , with div $u_{0}=0$ and $\theta_{0} \in W^{1, q}(\Omega)$. Assume that $\nu: \mathbb{R} \rightarrow(0, \infty)$ satisfies $0<\nu_{0} \leq \nu$, $\nu \in C^{1}(\mathbb{R})$ with $\nu^{\prime}(0)=0$ and

$$
\left|\nu^{\prime}(a)-\nu^{\prime}(b)\right| \leq k_{1}|a-b|, \text { for all } a, b \in \mathbb{R},
$$

where $\nu_{0}, k_{1}$ are positive constants. Moreover, suppose that $\nu(x)=k_{2}+\tilde{\nu}(x)$ with $k_{2}$ a positive constant and

$$
\tilde{\nu}(\omega) \in C\left([0, T] ; W^{1, q}(\Omega)\right) \text { when } \omega \in C\left([0, T] ; W^{1, q}(\Omega)\right) .
$$

Then there is $0<T^{\prime} \leq T$ and a unique solution $(u, \nabla \pi, \theta) \in \mathcal{E}_{p, T^{\prime}}^{2,1} \times L^{p}\left(Q_{T^{\prime}}\right) \times$ $C\left(\left[0, T^{\prime}\right] ; W^{1, q}(\Omega)\right)$ for (1.2)-(1.3) in $Q_{T^{\prime}}$. Moreover, there exists a positive constant $L_{0}$ such that if $\|f\|_{L^{p}\left(Q_{T}\right)}+\left\|u_{0}\right\|_{W^{2-\frac{2}{p}, p}(\Omega)} \leq L_{0}$, then $T^{\prime}=T$.

REMARK 1.1.

1. In Theorem 1.1, the condition (1.6) does not imply $\nu(\theta(x, t)) \in$ $C\left([0, T] ; W^{1, q}(\Omega)\right)$ when $\Omega$ is unbounded, because $k_{2}$ is a positive constant. Notice that it is compatible with the restriction $0<\nu_{0} \leq \nu$ and is necessary in order to apply a result of Abels in [2].

2. In several applications, it is common to consider the gravitational force parallel to the $x_{N}$-coordinate when $\Omega$ is a bounded domain $\Omega$, that is, $f=-g_{0} \vec{e}_{N}$. This approximation is covered in Theorem 1.1. For an exterior domain $\Omega$ with $0 \notin \Omega$, we can take $f(x)=g_{0} \nabla(1 /|x|)$ where $g_{0}>0$ is the gravitational constant. In fact, in this case we have $f(x) \in L^{(N / 2, \infty)}(\Omega) \cap L^{\infty}(\Omega) \subset L^{p}(\Omega)$, for $p \in(N / 2, \infty)$, and then $\|f\|_{L^{p}\left(Q_{T}\right)}=T^{1 / p}\|f(x)\|_{L^{p}(\Omega)}$. From a physical viewpoint, this case can be regarded as a version of the Bénard problem in an exterior domain. 
We also investigate whether the system (1.2)-(1.3) can be described as the limit of the fully viscous Boussinesq system, when the heat conductivity coefficient $\kappa$ goes to zero. For that matter, we first establish an existence result of strong solutions for the following full viscous Boussinesq system:

$$
\left\{\begin{aligned}
\partial_{t} u-\operatorname{div}\left(\nu(\theta)\left(\nabla u+\nabla^{*} u\right)\right)+u \cdot \nabla u+\nabla \pi & =\theta f \text { in } Q_{T}, \\
\operatorname{div} u & =0 \text { in } Q_{T}, \\
\partial_{t} \theta-\operatorname{div}(\kappa(\theta)(\nabla \theta))+u \cdot \nabla \theta & =g \text { in } Q_{T},
\end{aligned}\right.
$$

where $\kappa$ depends on temperature and satisfies $0 \leq \kappa(\theta) \leq \tilde{\kappa}$, for some positive constant $\tilde{\kappa}$. System (1.7) is completed with the following initial and boundary conditions:

$$
\left\{\begin{aligned}
\left.(u, \theta)\right|_{t=0} & =\left(u_{0}, \theta_{0}\right) \text { in } \Omega \\
u, \theta & =0 \text { on } \Gamma_{T}:=\partial \Omega \times(0, T) .
\end{aligned}\right.
$$

Proposition 1.2. In addition to the assumptions of Theorem 1.1, let $g \in L^{p}\left(Q_{T}\right)$ and $\left(u_{0}, \theta_{0}\right) \in W^{2-\frac{2}{p}, p}(\Omega) \times W^{2-\frac{2}{p}, p}(\Omega)$, div $u_{0}=0$ and $\left.\left(u_{0}, \theta_{0}\right)\right|_{\partial \Omega}=(0,0)$. Then there is $0<T^{\prime} \leq T$ such that (1.7)-(1.8) has a unique strong solution $(u, \nabla \pi, \theta) \in \mathcal{E}_{p, T^{\prime}}^{2,1} \times$ $L^{p}\left(Q_{T^{\prime}}\right) \times \mathcal{E}_{p, T^{\prime}}^{2,1}$.

In the next theorem we analyze the heat conductivity vanishing problem for (1.7)(1.8) in the $W^{1, p} \times W^{1, q}$-framework.

TheOREm 1.3. Under the hypotheses of Theorem 1.1. Assume further that $\Omega$ is a bounded domain and $\theta_{0} \in W^{2-\frac{2}{p}, p}(\Omega)$. Let $\left(u_{1}, \nabla \pi_{1}, \theta_{1}\right)$ be the local strong solution of (1.2)-(1.3) given by Theorem 1.1 and let $\left(u_{2}, \nabla \pi_{2}, \theta_{2}\right)$ be the local strong solution of (1.7)-(1.8) with $0 \leq \kappa(\theta) \leq \tilde{\kappa}$ and $g=0$ established in Proposition 1.2. There exists a common existence time interval $\left[0, T^{\prime}\right]$ for $\left(u_{1}, \nabla \pi_{1}, \theta_{1}\right)$ and $\left(u_{2}, \nabla \pi_{2}, \theta_{2}\right)$, such that $\left(u_{2}, \theta_{2}\right)$ converges to $\left(u_{1}, \theta_{1}\right)$ as $\tilde{\kappa} \rightarrow 0$ in $C\left(\left[0, T^{\prime}\right] ; W^{1, p}(\Omega) \times W^{1, q}(\Omega)\right)$.

\section{Linear problems}

2.1. Transport equation. We start by recalling a result about existence of solutions for a transport equation connected to (1.2).

Lemma 2.1. ([9, 21]) Let $u \in L^{1}\left(0, T ; W^{1, \infty}(\Omega)\right)$ with div $u=0$ and $\left.u\right|_{\partial \Omega}=0$. Then, for all $\theta_{0} \in W^{1, q}(\Omega)$ with $q \in[1, \infty)$, there exists a unique solution $\theta \in C\left([0, T] ; W^{1, q}(\Omega)\right)$ of the transport equation

$$
\left\{\begin{array}{r}
\partial_{t} \theta+u \cdot \nabla \theta=0 \text { in } Q_{T} \\
\left.\theta\right|_{t=0}=\theta_{0} \text { in } \Omega .
\end{array}\right.
$$

Moreover, $\theta$ satisfies the following estimate:

$$
\|\theta(t)\|_{W^{1, q}(\Omega)} \leq\left\|\theta_{0}\right\|_{W^{1, q}(\Omega)} e^{\int_{0}^{t}\|\nabla u(s)\|_{L^{\infty}(\Omega)} d s},
$$

for all $t \in[0, T]$.

The following lemma establishes a stability result of solutions for (2.1) with respect to the velocity field $u$. In [12] the reader can find a stability result for renormalized solutions in a weaker framework. 
Lemma 2.2. Assume that $N<p<\infty$ and $1 \leq q \leq \infty$. Let $\left\{u^{n}\right\}_{n \in \mathbb{N}}$ be a sequence in $\mathcal{E}_{p, T}^{2,1}$ satisfying div $u^{n}=0$ and $\left.u_{n}\right|_{\Gamma_{T}}=0$, and let $\theta^{n} \in L^{\infty}\left(0, T ; W^{1, q}(\Omega)\right)$ be the solution of (2.1) with initial data $\theta_{0} \in W^{1, q}(\Omega)$ and velocity field $u^{n}$. If $u^{n}$ converges to $u$ in $L^{1}\left(0, T ; W^{1, \infty}(\Omega)\right)$, then $\theta^{n}$ converges to $\theta$ in $L^{\infty}\left(0, T ; W^{1, q}(\Omega)\right)$, where $\theta$ is the solution of (2.1) with initial data $\theta_{0} \in W^{1, q}(\Omega)$ and velocity field $u$.

Proof. The difference $\left(u^{n}, \theta^{n}\right)-(u, \theta)$ satisfies the equation

$$
\left\{\begin{aligned}
\partial_{t}\left(\theta^{n}-\theta\right)+u^{n} \cdot \nabla\left(\theta^{n}-\theta\right) & =-\left(u^{n}-u\right) \cdot \nabla \theta \text { in } Q_{T}, \\
\left.\left(\theta^{n}-\theta\right)\right|_{t=0} & =0 \text { in } \Omega .
\end{aligned}\right.
$$

Let us denote by $\tilde{\psi}$ the flow of the field $u_{n}$, that is,

$$
\frac{\partial \tilde{\psi}(\tau, t, x)}{\partial \tau}=u_{n}(\tilde{\psi}(\tau, t, x), \tau),\left.\tilde{\psi}\right|_{\tau=t}=x .
$$

Because $\left.\left(\theta^{n}-\theta\right)\right|_{t=0}=0$, the equation $(2.3)_{1}$ implies

$$
\left(\theta^{n}-\theta\right)(x, t)=-\int_{0}^{t}\left(u^{n}-u\right) \cdot \nabla \theta\left(\tilde{\psi}\left(s, \tilde{\psi}(s, x)^{-1}\right)\right) d s .
$$

Computing the $L^{q}$-norm in (2.5) and afterwards employing the Hölder inequality, we obtain

$$
\begin{aligned}
\left\|\theta^{n}(t)-\theta(t)\right\|_{L^{q}(\Omega)} & \leq \int_{0}^{t}\left\|\left(u^{n}-u\right) \cdot \nabla \theta\right\|_{L^{q}(\Omega)} d s \\
& \leq\left\|u^{n}-u\right\|_{L^{1}\left(0, T ; L^{\infty}(\Omega)\right)}\|\nabla \theta\|_{L^{\infty}\left(0, T ; L^{q}(\Omega)\right)} \\
& \leq c\left\|u^{n}-u\right\|_{L^{1}\left(0, T ; L^{\infty}(\Omega)\right)} .
\end{aligned}
$$

Because $u^{n} \rightarrow u$ in $L^{1}\left(0, T ; W^{1, \infty}(\Omega)\right)$, the right hand side of (2.6) goes to zero, and then

$$
\theta^{n} \rightarrow \theta \text { in } L^{\infty}\left(0, T ; L^{q}(\Omega)\right)
$$

Now we compute the norm $L^{\infty}\left(0, T ; L^{q}(\Omega)\right)$ of the difference $\nabla \theta^{n}-\nabla \theta$. For $n \in \mathbb{N}$, let us take $\varrho_{n} \in C_{0}^{1}(\bar{\Omega})$ such that $\left\|\varrho_{n}-\theta_{0}\right\|_{W^{1, q}(\Omega)}<1 / n$. The solution $\theta_{\varrho_{n}} \in C^{1}\left(\bar{Q}_{T}\right)$ of

$$
\left\{\begin{aligned}
\partial_{t} \theta_{\varrho_{n}}+u \cdot \nabla \theta_{\varrho_{n}} & =0 \text { in } Q_{T} \\
\left.\theta\right|_{t=0} & =\varrho_{n} \text { in } \Omega
\end{aligned}\right.
$$

is given by the formula

$$
\theta_{\varrho_{n}}(x, t)=\varrho_{n}(\psi(0, t, x)),
$$

where $\psi(\tau, t, x)$ is the solution of the Cauchy problem

$$
\frac{\partial \psi(\tau, t, x)}{\partial \tau}=u(\psi(\tau, t, x), \tau),\left.\psi\right|_{\tau=t}=x,
$$

and $t$ is an arbitrary number in $[0, T]$ (see [21]). Due to the linearity of the problem (2.1) and estimate (2.2), we have

$$
\left\|\theta_{\varrho_{n}}(t)-\theta(t)\right\|_{L^{\infty}\left(0, T ; W^{1, q}(\Omega)\right)} \leq\left\|\varrho_{n}-\theta_{0}\right\|_{W^{1, q}(\Omega)} e^{\int_{0}^{t}\|\nabla u(s)\|_{L^{\infty}(\Omega)} d s},
$$


for all $t \in[0, T]$. Similarly, the solution $\theta_{\varrho_{n}}^{n}$ for

$$
\left\{\begin{array}{r}
\partial_{t} \theta_{\varrho_{n}}^{n}+u^{n} \cdot \nabla \theta_{\varrho_{n}}^{n}=0 \text { in } Q_{T} \\
\left.\theta\right|_{t=0}=\varrho_{n} \text { in } \Omega
\end{array}\right.
$$

belongs to $C^{1}\left(\bar{Q}_{T}\right)$ and is given by

$$
\theta_{\varrho}^{n}(x, t)=\varrho_{n}\left(\psi^{n}(0, t, x)\right),
$$

where $\psi^{n}(\tau, t, x)$ is the solution of the Cauchy problem

$$
\frac{\partial \psi^{n}(\tau, t, x)}{\partial \tau}=u^{n}\left(\psi^{n}(\tau, t, x), \tau\right),\left.\psi^{n}\right|_{\tau=t}=x .
$$

Furthermore

$$
\left\|\theta_{\varrho_{n}}^{n}(t)-\theta^{n}(t)\right\|_{L^{\infty}\left(0, T ; W^{1, q}(\Omega)\right)} \leq\left\|\varrho_{n}-\theta_{0}\right\|_{W^{1, q}(\Omega)} e^{\int_{0}^{t}\left\|\nabla u^{n}(s)\right\|_{L^{\infty}(\Omega)} d s},
$$

for all $t \in[0, T]$. Notice that the map $x \mapsto \psi^{n}(\tau, t, x)$, where $\psi^{n}$ is the solution of (2.11), has Jacobian equal to one.

Computing the difference $\partial \theta_{\varrho_{n}}^{n} / \partial x_{i}-\partial \theta_{\varrho_{n}} / \partial x_{i}$, we have the equality

$$
\begin{array}{r}
\frac{\partial \theta_{\varrho_{n}}^{n}}{\partial x_{i}}-\frac{\partial \theta_{\varrho_{n}}}{\partial x_{i}}=\sum_{k=1}^{n}\left[\frac{\partial \varrho_{n}}{\partial x_{k}}\left(\psi^{n}(0, t, x)\right)-\frac{\partial \varrho_{n}}{\partial x_{k}}(\psi(0, t, x))\right] \frac{\partial \psi_{k}^{n}}{\partial x_{i}}(0, t, x) \\
-\sum_{k=1}^{n} \frac{\partial \varrho_{n}}{\partial x_{k}}(\psi(0, t, x))\left[\frac{\partial \psi_{k}}{\partial x_{i}}(0, t, x)-\frac{\partial \psi_{k}^{n}}{\partial x_{i}}(0, t, x)\right] .
\end{array}
$$

Therefore

$$
\begin{aligned}
&\left\|\nabla\left(\theta_{\varrho_{n}}^{n}-\theta_{\varrho_{n}}\right)\right\|_{L^{\infty}\left(0, T ; L^{q}(\Omega)\right)} \leq c\left\|\nabla \varrho_{n}\right\|_{L^{q}(\Omega)}\left\|\nabla \psi^{n}(0, t, x)-\nabla \psi(0, t, x)\right\|_{L^{\infty}\left(Q_{T}\right)} \\
&++c\left\|\nabla \psi^{n}(0, t, x)\right\|_{L^{\infty}\left(Q_{T}\right)} \\
& \times\left\|\nabla \varrho_{n}\left(\psi^{n}(0, t, x)\right)-\nabla \varrho_{n}(\psi(0, t, x))\right\|_{L^{\infty}\left(0, T ; L^{q}(\Omega)\right)} .
\end{aligned}
$$

It follows from (2.8) and (2.11) that

$$
\left\{\begin{array}{c}
\psi(\tau, t, x)=x-\int_{\tau}^{t} u(\psi(s, t, x), s) d s \\
\psi^{n}(\tau, t, x)=x-\int_{\tau}^{t} u^{n}\left(\psi^{n}(s, t, x), s\right) d s
\end{array}\right.
$$

Then, for $\tau \leq t$, we get

$$
\begin{aligned}
\left|\psi^{n}(\tau, t, x)-\psi(\tau, t, x)\right| & \leq \int_{\tau}^{t}\left|u^{n}\left(\psi^{n}(s, t, x), s\right)-u(\psi(s, t, x), s)\right| d s \\
& \leq \int_{\tau}^{t}\left|u^{n}\left(\psi^{n}(s, t, x), s\right)-u^{n}(\psi(s, t, x), s)\right| d s \\
& \quad+\int_{\tau}^{t}\left|u^{n}(\psi(s, t, x), s)-u(\psi(s, t, x), s)\right| d s .
\end{aligned}
$$


Observe that

$$
\left|u^{n}\left(\psi^{n}(s, t, x), s\right)-u^{n}(\psi(s, t, x), s)\right| \leq\left\|\nabla u^{n}(s)\right\|_{L^{\infty}(\Omega)}\left|\psi^{n}(s, t, x)-\psi(s, t, x)\right|,
$$

for almost every $s \in(0, T)$. The inequalities (2.15) and (2.16) imply

$$
\begin{aligned}
\left|\psi^{n}(\tau, t, x)-\psi(\tau, t, x)\right| \leq \int_{\tau}^{t} & \left\|\nabla u^{n}(s)\right\|_{L^{\infty}(\Omega)}\left|\psi^{n}(s, t, x)-\psi(s, t, x)\right| d s \\
& +\int_{\tau}^{t}\left|u^{n}(\psi(s, t, x), s)-u(\psi(s, t, x), s)\right| d s .
\end{aligned}
$$

In view of (2.17), we can use the Gronwall inequality to obtain

$$
\left|\psi^{n}(\tau, t, x)-\psi(\tau, t, x)\right| \leq c e^{\left\|\nabla u^{n}\right\|_{L^{1}\left(0, T ; L^{\infty}(\Omega)\right)}} \times\left\|u^{n}-u\right\|_{L^{1}\left(0, T ; L^{\infty}(\Omega)\right)} .
$$

Taking $\tau=0$ in the last inequality we get

$$
\begin{aligned}
\left\|\psi^{n}(0, t, x)-\psi(0, t, x)\right\|_{L^{\infty}\left(Q_{T}\right)} & \leq c e^{\left\|\nabla u^{n}\right\|_{L^{1}\left(0, T ; L^{\infty}(\Omega)\right)} \times\left\|u^{n}-u\right\|_{L^{1}\left(0, T ; L^{\infty}(\Omega)\right)}} \\
& \rightarrow 0, \text { as } n \rightarrow \infty
\end{aligned}
$$

From (2.18) and the regularity of $\varrho_{n}$, we can conclude that the second term in the right hand side of (2.13) goes to zero as $n \rightarrow \infty$.

Now we analyze the first one in the right hand side of (2.13). We have from (2.14) that

$$
\begin{aligned}
\frac{\partial \psi^{n}(\tau)}{\partial x_{i}}-\frac{\partial \psi(\tau)}{\partial x_{i}}=- & \sum_{k=1}^{n} \int_{\tau}^{t} \frac{\partial u^{n}(\tau)}{\partial x_{k}}\left(\psi^{n}(s, t, x), s\right) \frac{\partial \psi^{n}(s, t, x)}{\partial x_{i}} d s \\
& +\sum_{k=1}^{n} \int_{\tau}^{t} \frac{\partial u(\tau)}{\partial x_{k}}(\psi(s, t, x), s) \frac{\partial \psi(s, t, x)}{\partial x_{i}} d s \\
=- & \sum_{k=1}^{n} \int_{\tau}^{t}\left[\frac{\partial u^{n}(\tau)}{\partial x_{k}}\left(\psi^{n}(s, t, x), s\right)-\frac{\partial u(\tau)}{\partial x_{k}}(\psi(s, t, x), s)\right] \frac{\partial \psi^{n}(s, t, x)}{\partial x_{i}} d s \\
& +\sum_{k=1}^{n} \int_{\tau}^{t} \frac{\partial u(\tau)}{\partial x_{k}}(\psi(s, t, x), s)\left[\frac{\partial \psi(s, t, x)}{\partial x_{i}}-\frac{\partial \psi^{n}(s, t, x)}{\partial x_{i}}\right] d s
\end{aligned}
$$

Therefore

$$
\begin{aligned}
\left|\nabla \psi^{n}(\tau)-\nabla \psi(\tau)\right| \leq c \int_{\tau}^{t} & \left\|\nabla \psi^{n}(s)\right\|_{L^{\infty}\left(Q_{T}\right)}\left\|\nabla u^{n}(s)-\nabla u(s)\right\|_{L^{\infty}(\Omega)} d s \\
& +c \int_{\tau}^{t}\|\nabla u(s)\|_{L^{\infty}(\Omega)}\left|\nabla \psi^{n}(s)-\nabla \psi(s)\right| d s .
\end{aligned}
$$

Using the Gronwall inequality, it follows that

$$
\begin{aligned}
& \left\|\nabla \psi^{n}(t)-\nabla \psi(t)\right\|_{L^{\infty}\left(Q_{T}\right)} \leq c e^{\left.\|u\|_{L^{1}\left(0, T ; W^{1}, \infty\right.}(\Omega)\right)} \\
& \times \int_{0}^{t}\left\|\nabla \psi^{n}(s)\right\|_{L^{\infty}\left(Q_{T}\right)}\left\|\nabla u^{n}(s)-\nabla u(s)\right\|_{L^{\infty}(\Omega)} d s \\
& \leq c e^{\|u\|_{L^{1}\left(0, T ; W^{1, \infty}(\Omega)\right)}}\left\|u^{n}-u\right\|_{L^{1}\left(0, T ; W^{1, \infty}(\Omega)\right)}\left\|\nabla \psi^{n}(s)\right\|_{L^{\infty}\left(Q_{T}\right)} \\
& \leq c T e^{c T\|\nabla u\|_{L^{\infty}\left(Q_{T}\right)}}\left\|u^{n}-u\right\|_{L^{1}\left(0, T ; W^{1, \infty}(\Omega)\right)} .
\end{aligned}
$$


Because $u^{n} \rightarrow u$ in $L^{1}\left(0, T ; W^{1, \infty}(\Omega)\right)$, the first term of the right hand side of (2.13) tends to zero as $n \rightarrow \infty$. Then, from the triangle inequality, (2.9), (2.12), and (2.13), we obtain

$$
\begin{gathered}
\left\|\nabla \theta^{n}-\nabla \theta\right\|_{L^{\infty}\left(0, T ; L^{q}(\Omega)\right)} \leq\left\|\nabla \theta^{n}-\nabla \theta_{\varrho_{n}}^{n}\right\|_{L^{\infty}\left(0, T ; L^{q}(\Omega)\right)}+\left\|\nabla \theta_{\varrho_{n}}^{n}-\nabla \theta \varrho_{n}\right\|_{L^{\infty}\left(0, T ; L^{q}(\Omega)\right)} \\
+\left\|\nabla \theta_{\varrho_{n}}-\nabla \theta\right\|_{L^{\infty}\left(0, T ; L^{q}(\Omega)\right)} \rightarrow 0, \text { as } n \rightarrow \infty .
\end{gathered}
$$

\subsection{Nonstationary Stokes equation with variable viscosity.}

Lemma 2.3. Let $N<p \leq q<\infty, \theta: Q_{T} \rightarrow \mathbb{R}$, and $\nu: \mathbb{R} \rightarrow(0, \infty)$ be such that

$$
0<\nu_{0} \leq \nu(\theta)=k_{2}+\tilde{\nu}(\theta) \text { with } \tilde{\nu}(\theta) \in C\left([0, T] ; W^{1, q}(\Omega)\right),
$$

where $\nu_{0}$ and $k_{2}$ are positive constants. Assume also that $h \in L^{p}\left(Q_{T}\right)$ and $u_{0} \in$ $W^{2-\frac{2}{p}, p}(\Omega)$ with $\left.u_{0}\right|_{\partial \Omega}=0$ and div $u_{0}=0$. Then there exists a unique solution $(u, \nabla \pi) \in$ $\mathcal{E}_{p, T}^{2,1} \times L^{p}\left(Q_{T}\right)$ for

$$
\left\{\begin{aligned}
\partial_{t} u-\operatorname{div}\left(\nu(\theta(x, t))\left(\nabla u+\nabla^{*} u\right)\right)+\nabla \pi & =h \text { in } Q_{T}, \\
\operatorname{div} u & =0 \text { in } Q_{T} \\
u & =0 \text { on } \Gamma_{T} \\
\left.u\right|_{t=0} & =u_{0} \text { in } \Omega
\end{aligned}\right.
$$

Moreover,

$$
\|u\|_{\mathcal{E}_{p, T}^{2,1}}+\|\pi\|_{\mathcal{E}_{p, T}^{1,0}} \leq \tilde{C}\left(\|h\|_{L^{p}\left(Q_{T}\right)}+\left\|u_{0}\right\|_{W^{2-\frac{2}{p}, p}(\Omega)}\right) .
$$

The constant $\tilde{C}>0$ depends continuously on $\delta \in[0, \infty)$, where $\delta=$ $\|\tilde{\nu}(\theta)\|_{L^{\infty}\left(0, T ; W^{1, q}(\Omega)\right)}$. The dependence of $\tilde{C}$ on $T$ is given only by means of the norm $\|\tilde{\nu}(\theta)\|_{L^{\infty}\left(0, T ; W^{1, q}(\Omega)\right)}$.

Proof. First notice that $C\left([0, T] ; W^{1, q}(\Omega)\right)=B C U\left([0, T] ; W^{1, q}(\Omega)\right)$, because the interval considered, $[0, T]$, is compact. Second, recall that the results of [2] cover both smooth bounded and exterior domains $\Omega$. Therefore we have that $0<\nu_{0} \leq \nu(\theta(x, t))=$ $k_{2}+\tilde{\nu}(\theta(x, t))$ with $\tilde{\nu}(\theta(x, t)) \in B C U\left([0, T] ; W^{1, q}(\Omega)\right)$, and then the proof of Lemma 2.3 follows as a particular case of [2, Theorem 1.1]. Observe that in our case we can remove the condition $p \neq 3$ in [2, Theorem 1.1] because we are considering a pure Dirichlet boundary condition (see item 1 of Lemma 2.4 in [2]).

\section{Proof of Theorem 1.1}

\subsection{Existence of strong solutions.}

Proof. First step. We prove the existence of strong solutions for the problem (1.2)-(1.3) by using successive approximations. Given $u^{0}=u_{0}$ and $\theta^{0}=\theta_{0}$, we define recursively $u^{m}, \pi^{m}, \theta^{m}$, for $m \in \mathbb{N}$, as the solution of the following system:

$$
\left\{\begin{aligned}
\partial_{t} u^{m+1}-\operatorname{div}\left(\nu\left(\theta^{m+1}\right)\left(\nabla u^{m+1}+\nabla^{*} u^{m+1}\right)\right)+\nabla \pi^{m+1} & =\theta^{m+1} f-u^{m} \cdot \nabla u^{m} \text { in } Q_{T}, \\
\operatorname{div} u^{m+1} & =0 \text { in } Q_{T}, \\
\partial_{t} \theta^{m+1}+u^{m} \cdot \nabla \theta^{m+1} & =0 \text { in } Q_{T}, \\
u^{m+1} & =0 \text { on } \Gamma_{T}, \\
\left.\left(u^{m+1}, \theta^{m+1}\right)\right|_{t=0} & =\left(u_{0}, \theta_{0}\right) \text { in } \Omega .
\end{aligned}\right.
$$


Because $u^{m} \in \mathcal{E}_{p, T}^{2,1}$ and $p>N$, it follows that $u^{m} \in L^{1}\left(0, T ; W^{1, \infty}(\Omega)\right)$. Since $\operatorname{div}\left(u^{m}\right)=$ 0 , from Lemma 2.1, there exists $\theta^{m+1} \in C\left([0, T] ; W^{1, q}(\Omega)\right) \subset L^{\infty}\left(Q_{T}\right)$ such that

$$
\left\{\begin{aligned}
\partial_{t} \theta^{m+1}+u^{m} \cdot \nabla \theta^{m+1} & =0 \text { in } Q_{T} \\
\left.\theta^{m+1}\right|_{t=0} & =\theta_{0} \text { in } \Omega .
\end{aligned}\right.
$$

We have that $\theta^{m+1} f \in L^{p}\left(Q_{T}\right)$ for all $m \in \mathbb{N}$. Moreover, the inclusion $\mathcal{E}_{p, T}^{2,1} \subset L^{\infty}\left(Q_{T}\right)$ implies $u^{m} \cdot \nabla u^{m} \in L^{p}\left(Q_{T}\right)$. In view of $\theta^{m+1} \in C\left([0, T] ; W^{1, q}(\Omega)\right)$, we can apply Lemma 2.3 in order to obtain the existence of a unique solution $\left(u^{m}, \nabla \pi^{m}\right) \in \mathcal{E}_{p, T}^{2,1} \times L^{p}\left(Q_{T}\right)$ for $(3.1)_{1}-(3.1)_{2}$. Thus, we get a sequence $\left(u^{m}, \nabla \pi^{m}, \theta^{m}\right)$ whose elements satisfy (3.1) and belong to the class

$$
\mathcal{E}_{p, T}^{2,1} \times L^{p}\left(Q_{T}\right) \times C\left([0, T] ; W^{1, q}(\Omega)\right) .
$$

In the sequel we obtain suitable uniform estimates for $\left(u^{m}, \nabla \pi^{m}, \theta^{m}\right)$. Using that $\theta^{m+1} \in L^{\infty}\left(0, T ; W^{1, q}(\Omega)\right) \subset L^{\infty}\left(Q_{T}\right)$, we get from (2.20) that

$$
\begin{gathered}
\left\|u^{m+1}\right\|_{\mathcal{E}_{p, T}^{2,1}}+\left\|\nabla \pi^{m+1}\right\|_{L^{p}\left(Q_{T}\right)} \leq \tilde{C}\left(\left\|u_{0}\right\|_{W^{2-\frac{2}{p}, p}(\Omega)}\right) \\
+\tilde{C}\left(\left\|\theta^{m+1} f\right\|_{L^{p}\left(Q_{T}\right)}+\left\|u^{m} \cdot \nabla u^{m}\right\|_{L^{p}\left(Q_{T}\right)}\right) \\
\leq \tilde{C}\left(\left\|u_{0}\right\|_{W^{2-\frac{2}{p}, p}(\Omega)}+\left\|\theta^{m+1}\right\|_{L^{\infty}\left(Q_{T}\right)}\|f\|_{L^{p}\left(Q_{T}\right)}\right. \\
\left.+\left\|u^{m} \cdot \nabla u^{m}\right\|_{L^{p}\left(Q_{T}\right)}\right) .
\end{gathered}
$$

Notice also that

$$
\left\|u^{m} \cdot \nabla u^{m}\right\|_{L^{p}\left(Q_{T}\right)} \leq c\left\|u^{m}\right\|_{L^{\infty}\left(Q_{T}\right)}\left\|\nabla u^{m}\right\|_{L^{p}\left(Q_{T}\right)} .
$$

Moreover, for $a=(p-N) /(2 p-N)<1$, we have the inequalities

$$
\left\|\nabla u^{m}\right\|_{L^{p}(\Omega)} \leq\left\|u^{m}\right\|_{W^{1, p}(\Omega)} \leq c\left\|u^{m}\right\|_{W^{2, p}(\Omega)}^{a}\left\|u^{m}\right\|_{L^{\infty}(\Omega)}^{1-a} .
$$

Then

$$
\int_{0}^{T}\left\|\nabla u^{m}\right\|_{L^{p}(\Omega)}^{p} d t \leq c\left\|u^{m}\right\|_{L^{\infty}\left(Q_{T}\right)}^{(1-a) p} \int_{0}^{T}\left\|u^{m}\right\|_{W^{2, p}(\Omega)}^{a p} d t .
$$

Now it follows from the Hölder inequality that

$$
\begin{aligned}
\int_{0}^{T}\left\|u^{m}\right\|_{W^{2, p}(\Omega)}^{a p} d t & \leq\left(\int_{0}^{T} 1 d t\right)^{1-a}\left(\int_{0}^{T}\left\|u^{m}(t)\right\|_{W^{2, p}(\Omega)}^{p} d t\right)^{a} \\
& \leq T^{1-a}\left\|u^{m}\right\|_{\mathcal{E}_{p, T}^{a p}}^{a, 1} \\
& \leq T^{1-a}\left\{\left\|u^{m}\right\|_{\mathcal{E}_{p, T}^{2,1}}+\left\|\nabla \pi^{m}\right\|_{L^{p}\left(Q_{T}\right)}\right\}^{a p}
\end{aligned}
$$

Using the estimate (3.5) we get

$$
\begin{aligned}
\left\|\nabla u^{m}\right\|_{L^{p}\left(Q_{T}\right)} & \leq c\left\{\left\|u^{m}\right\|_{\mathcal{E}_{p, T}^{2,1}}+\left\|\nabla \pi^{m}\right\|_{L^{p}\left(Q_{T}\right)}\right\}^{1-a} T^{\frac{1-a}{p}}\left\{\left\|u^{m}\right\|_{\mathcal{E}_{p, T}^{2,1}}+\left\|\nabla \pi^{m}\right\|_{L^{p}\left(Q_{T}\right)}\right\}^{a} \\
& \leq c T^{\frac{1-a}{p}}\left\{\left\|u^{m}\right\|_{\mathcal{E}_{p, T}^{2,1}}+\left\|\nabla \pi^{m}\right\|_{L^{p}\left(Q_{T}\right)}\right\}
\end{aligned}
$$


which, together with (3.3), yields

$$
\begin{aligned}
\left\|u^{m} \cdot \nabla u^{m}\right\|_{L^{p}\left(Q_{T}\right)} & \leq c\left\|u^{m}\right\|_{L^{\infty}\left(Q_{T}\right)} T^{\frac{1-a}{p}}\left\{\left\|u^{m}\right\|_{\mathcal{E}_{p, T}^{2,1}}+\left\|\nabla \pi^{m}\right\|_{L^{p}\left(Q_{T}\right)}\right\} \\
& \leq c T^{\frac{1-a}{p}}\left\{\left\|u^{m}\right\|_{\mathcal{E}_{p, T}^{2,1}}+\left\|\nabla \pi^{m}\right\|_{L^{p}\left(Q_{T}\right)}\right\}^{2} .
\end{aligned}
$$

On the other hand, from estimate (2.2) we have

$$
\left\|\theta^{m+1}(t)\right\|_{W^{1, q}(\Omega)} \leq\left\|\theta_{0}\right\|_{W^{1, q}(\Omega)} e^{\int_{0}^{t}\left\|\nabla u^{m}(s)\right\|_{L^{\infty}(\Omega)} d s}, \forall t \in[0, T], m=1,2, \ldots
$$

Let us now denote

$$
\Psi^{m}(t):=\left\|u^{m}\right\|_{\mathcal{E}_{p}^{2,1}\left(Q_{t}\right)}+\left\|\nabla \pi^{m}\right\|_{L^{p}\left(Q_{t}\right)}, t \in[0, T] .
$$

Coming back to the inequality (3.2), taking into account (3.8)-(3.9), and using Young's inequality, we can estimate

$$
\begin{aligned}
\Psi^{m+1}(t) & \leq \tilde{C}\left(\left\|u_{0}\right\|_{W^{2-\frac{2}{p}, p}(\Omega)}+\left\|\theta^{m+1}\right\|_{L^{\infty}\left(Q_{t}\right)}\|f\|_{L^{p}\left(Q_{T}\right)}+\left\|u^{m} \cdot \nabla u^{m}\right\|_{L^{p}\left(Q_{t}\right)}\right) \\
& \leq \tilde{C}\left(\left\|u_{0}\right\|_{W^{2-\frac{2}{p}, p}(\Omega)}+\left\|\theta_{0}\right\|_{W^{1, q}(\Omega)} e^{\int_{0}^{t}\left\|\nabla u^{m}(s)\right\|_{L^{\infty}(\Omega)} d s}\|f\|_{L^{p}\left(Q_{T}\right)}+t^{\frac{1-a}{p}} \Psi^{m}(t)^{2}\right) \\
& \leq \tilde{C}\left(\left\|u_{0}\right\|_{W^{2-\frac{2}{p}, p}(\Omega)}+\left\|\theta_{0}\right\|_{W^{1, q}(\Omega)} e^{c_{1} t^{1 / p^{\prime}} \Psi^{m}(t)}\|f\|_{L^{p}\left(Q_{T}\right)}\right)+\tilde{C} t^{\frac{1-a}{p}} \Psi^{m}(t)^{2} \\
& \leq \tilde{C}\left(\left\|u_{0}\right\|_{W^{2-\frac{2}{p}, p}(\Omega)}+\left\|\theta_{0}\right\|_{W^{1, q}(\Omega)}\|f\|_{L^{p}\left(Q_{T}\right)}\right) e^{c_{1} t^{1 / p^{\prime}} \Psi^{m}(t)}+\tilde{C} t^{\frac{1-a}{p}} \Psi^{m}(t)^{2} .
\end{aligned}
$$

According to Lemma 2.3, the constant $\tilde{C}$ depends continuously on the norm of $\tilde{\nu}\left(\theta^{m}\right)$ in $C\left([0, T] ; W^{1, q}(\Omega)\right)$. Now, because $\theta^{m}$ satisfies the transport equation

$$
\partial_{t} \theta^{m+1}+u^{m} \cdot \nabla \theta^{m+1}=0 \text { in } Q_{T},\left.\theta^{m+1}\right|_{t=0}=\theta_{0},
$$

we have that $\tilde{\nu}\left(\theta^{m+1}\right)$ satisfies

$$
\partial_{t}\left(\tilde{\nu}\left(\theta^{m+1}\right)\right)+u^{m} \cdot \nabla\left(\tilde{\nu}\left(\theta^{m+1}\right)\right)=0 \text { in } Q_{T},\left.\tilde{\nu}\left(\theta^{m+1}\right)\right|_{t=0}=\tilde{\nu}\left(\theta_{0}\right) ;
$$

moreover, for all $t \in[0, T]$,

$$
\begin{aligned}
\left\|\tilde{\nu}\left(\theta^{m+1}\right)(t)\right\|_{W^{1, q}(\Omega)} & \leq\left\|\tilde{\nu}\left(\theta_{0}\right)\right\|_{W^{1, q}(\Omega)} e^{\int_{0}^{t}\left\|\nabla u^{m}(s)\right\|_{L^{\infty}(\Omega)} d s} \\
& \leq\left\|\tilde{\nu}\left(\theta_{0}\right)\right\|_{W^{1, q}(\Omega)} e^{c_{1} t^{1 / p^{\prime}} \Psi^{m}(t)} \\
& \leq c e^{c_{1} t^{1 / p^{\prime}} \Psi^{m}(t)}
\end{aligned}
$$

Assume that $\Psi^{m}\left(T^{\prime}\right) \leq 4 c A$, for $T^{\prime} \in(0, T]$, where $c$ is the absolute constant in (3.11), and $A>0$ is independent of $m$ and will be chosen later. It follows that

$$
\left\|\tilde{\nu}\left(\theta_{m}\right)\right\|_{L^{\infty}\left(0, t ; W^{1, q}(\Omega)\right)} \leq c e^{c_{1}\left(T^{\prime}\right)^{1 / p^{\prime}} 4 c A}, \text { for all } t \in\left[0, T^{\prime}\right]
$$

From (3.12) and the continuity of $\tilde{C}=\tilde{C}\left(\left\|\tilde{\nu}\left(\theta_{m}\right)\right\|_{L^{\infty}\left(0, t ; W^{1, q}(\Omega)\right)}\right)$ with respect to $\left\|\tilde{\nu}\left(\theta_{m}\right)\right\|_{L^{\infty}\left(0, t ; W^{1, q}(\Omega)\right)}$, we obtain that

$$
\tilde{C}=\tilde{C}\left(\left\|\tilde{\nu}\left(\theta_{m}\right)\right\|_{L^{\infty}\left(0, t ; W^{1, q}(\Omega)\right)}\right) \leq M, \text { for all } t \in\left[0, T^{\prime}\right],
$$


where $M>0$ is a constant independent of $m$.

Let

$$
A:=\left(\left\|u_{0}\right\|_{W^{2-\frac{2}{p}, p}(\Omega)}+\left\|\theta_{0}\right\|_{W^{1, q}(\Omega)}\|f\|_{L^{p}\left(Q_{T}\right)}\right)
$$

and take $T^{\prime} \in(0, T]$ small enough in a such way that

$$
4 c_{1} c\left(T^{\prime}\right)^{1 / p^{\prime}} A \leq \ln (2 c / M), 8 c A M\left(T^{\prime}\right)^{\frac{1-a}{p}} \leq 1 .
$$

If $\Psi^{m}\left(T^{\prime}\right) \leq 4 c A$ then it follows from (3.10) that $\Psi^{m+1}\left(T^{\prime}\right) \leq 4 c A$. Now an induction argument shows that $\left\{\Psi^{m}\left(T^{\prime}\right)\right\}_{m \geq 1}$ is a real bounded sequence, and so $u^{m}$ and $\nabla \pi^{m}$ are uniformly bounded in $\mathcal{E}_{p, T^{\prime}}^{2,1}$ and $L^{p}\left(Q_{T^{\prime}}\right)$, respectively. Moreover, the inequality (2.2) yields

$$
\begin{aligned}
\left\|\theta^{m}\right\|_{L^{\infty}\left(0, t ; W^{1, q}(\Omega)\right)} & \leq\left\|\theta_{0}\right\|_{W^{1, q}(\Omega)} e^{\int_{0}^{T^{\prime}}\left\|\nabla u^{m}(s)\right\|_{L^{\infty}(\Omega)} d s} \\
& \leq\left\|\theta_{0}\right\|_{W^{1, q}(\Omega)} e^{c_{1}\left(T^{\prime}\right)^{1 / p^{\prime}} \Psi^{m}\left(T^{\prime}\right)} \\
& \leq c\left\|\theta_{0}\right\|_{W^{1, q}(\Omega)}, \text { for all } t \in\left[0, T^{\prime}\right]
\end{aligned}
$$

and therefore $\theta^{m}$ is uniformly bounded in $C\left(\left[0, T^{\prime}\right] ; W^{1, q}(\Omega)\right)$.

Second step. In order to obtain the convergence of the sequence $\left\{u^{m}, \pi^{m}, \theta^{m}\right\}$, we compute the respective norms in the equation satisfied by the triple

$$
\left(u^{m+1}-u^{m}, \pi^{m+1}-\pi^{m}, \theta^{m+1}-\theta^{m}\right) .
$$

For that, we denote $U^{m}:=u^{m+1}-u^{m}, \Pi^{m}:=\pi^{m+1}-\pi^{m}$, and $\Theta^{m}:=\theta^{m+1}-\theta^{m}$. The triple $\left(U^{m}, \Pi^{m}, \Theta^{m}\right)$ satisfies the system

$$
\left\{\begin{array}{l}
\partial_{t} U^{m}-\operatorname{div}\left(\nu\left(\theta^{m+1}\right)\left(\nabla U^{m}+\nabla^{*} U^{m}\right)\right)+\nabla \Pi^{m+1} \\
=\Theta^{m} f-U^{m-1} \cdot \nabla u^{m}-u^{m-1} \cdot \nabla U^{m-1}+\operatorname{div}\left(\left(\nu\left(\theta^{m+1}\right)-\nu\left(\theta^{m}\right)\right)\left(\nabla u^{m}+\nabla^{*} u^{m}\right)\right) \\
\operatorname{div} U^{m}=0 \\
\partial_{t} \Theta^{m}+u^{m} \cdot \nabla \Theta^{m}=-U^{m-1} \cdot \nabla \theta^{m} \\
U^{m}=0 \text { on } \Gamma_{T}, \\
\left.\left(U^{m}, \Theta^{m}\right)\right|_{t=0}=(0,0) \text { in } \Omega .
\end{array}\right.
$$

Employing the inequality (2.20), we obtain

$$
\begin{aligned}
\left\|U^{m}\right\|_{\mathcal{E}_{p, t}^{2,1}}+\left\|\nabla \Pi^{m}\right\|_{L^{p}\left(Q_{t}\right)} \leq \tilde{C}\left(\left\|\Theta^{m} f\right\|_{L^{p}\left(Q_{t}\right)}+\left\|U^{m-1} \cdot \nabla u^{m}\right\|_{L^{p}\left(Q_{t}\right)}\right. & \\
& +\left\|\operatorname{div}\left(\nu\left(\theta^{m+1}\right)-\nu\left(\theta^{m}\right)\right)\left(\nabla u^{m}+\nabla^{*} u^{m}\right)\right\|_{L^{p}\left(Q_{t}\right)} \\
& \left.+\left\|u^{m-1} \cdot \nabla U^{m-1}\right\|_{L^{p}\left(Q_{t}\right)}\right):=\tilde{C}\left(I_{1}+I_{2}+I_{3}+I_{4}\right),
\end{aligned}
$$

for all $t \leq T^{\prime}$. Proceeding similarly to the proof of Lemma 2.2 (see (2.6)) with $u^{m+1}$ and $u^{m}$ in place of $u^{n}$ and $u$, respectively, one gets

$$
\left\|\Theta^{m}\right\|_{L^{\infty}\left(0, t ; W^{1, q}(\Omega)\right)} \leq c\left\|U^{m-1}\right\|_{L^{p}\left(0, t ; W^{2, p}(\Omega)\right)} .
$$

It follows from the Hölder inequality and (3.15) that

$$
I_{1} \leq\left\|\Theta^{m}\right\|_{L^{\infty}\left(Q_{t}\right)}\|f\|_{L^{p}\left(Q_{t}\right)} \leq c\left\|\Theta^{m}\right\|_{L^{\infty}\left(0, t ; W^{1, q}(\Omega)\right)} \leq c\left\|U^{m-1}\right\|_{L^{p}\left(0, t ; W^{2, p}(\Omega)\right)} .
$$


Moreover, recalling that $u^{m}, u^{m-1} \in \mathcal{E}_{p, T^{\prime}}^{2,1}$, we obtain from the Hölder inequality that

$$
I_{2} \leq\left\|U^{m-1}\right\|_{L^{p}\left(Q_{t}\right)}\left\|\nabla u^{m}\right\|_{L^{\infty}\left(Q_{t}\right)} \leq c\left\|U^{m-1}\right\|_{L^{p}\left(0, t ; W^{2, p}(\Omega)\right)}
$$

and

$$
I_{4} \leq\left\|U^{m-1}\right\|_{L^{p}\left(Q_{t}\right)}\left\|\nabla u^{m-1}\right\|_{L^{\infty}\left(Q_{t}\right)} \leq c\left\|U^{m-1}\right\|_{L^{p}\left(0, t ; W^{2, p}(\Omega)\right)} .
$$

Notice that

$$
\begin{aligned}
& \operatorname{div}\left(\left(\nu\left(\theta^{m+1}\right)-\nu\left(\theta^{m}\right)\right)\left(\nabla u^{m}+\nabla^{*} u^{m}\right)\right) \\
= & \left(\left(\nu\left(\theta^{m+1}\right)-\nu\left(\theta^{m}\right)\right) \nabla\left(\nabla u^{m}+\nabla^{*} u^{m}\right)\right. \\
& \quad+\left(\nu^{\prime}\left(\theta^{m+1}\right) \nabla \theta^{m+1}-\nu^{\prime}\left(\theta^{m}\right) \nabla \theta^{m}\right)\left(\nabla u^{m}+\nabla^{*} u^{m}\right) \\
= & \left(\nu\left(\theta^{m+1}\right)-\nu\left(\theta^{m}\right)\right) \nabla\left(\nabla u^{m}+\nabla^{*} u^{m}\right) \\
& \quad+\nu^{\prime}\left(\theta^{m+1}\right) \nabla \Theta^{m}+\left[\nu^{\prime}\left(\theta^{m+1}\right)-\nu^{\prime}\left(\theta^{m}\right)\right] \nabla \theta^{m} .
\end{aligned}
$$

The assumptions on $\nu$ yield

$$
|\nu(a)-\nu(b)| \leq\left(\sup _{d \in(a, b)}\left|\nu^{\prime}(d)\right|\right)|a-b| \leq k_{1}\left(\sup _{d \in(a, b)}|d|\right)|a-b| \leq k_{1}(|a|+|b|)|a-b| .
$$

Using Hölder's inequality, (3.19), and that $\left\|\theta^{m}\right\|_{L^{\infty}\left(Q_{T^{\prime}}\right)}$ is uniformly bounded, we can bound each term on the right hand side of (3.18) as follows:

$$
\begin{gathered}
\left\|\left(\nu\left(\theta^{m+1}\right)-\nu\left(\theta^{m}\right)\right) \nabla\left(\nabla u^{m}+\nabla^{*} u^{m}\right)\right\|_{L^{p}\left(Q_{t}\right)} \leq c\left(\left\|\theta^{m+1}\right\|_{L^{\infty}\left(Q_{T^{\prime}}\right)}+\left\|\theta^{m}\right\|_{L^{\infty}\left(Q_{T^{\prime}}\right)}\right) \\
\times\left\|\Theta^{m}\right\|_{L^{\infty}\left(Q_{t}\right)}\left\|u^{m}\right\|_{\mathcal{E}_{p, T^{\prime}}^{2,1}} \\
\leq c\left\|\Theta^{m}\right\|_{L^{\infty}\left(Q_{t}\right)}\left\|u^{m}\right\|_{\mathcal{E}_{p, T^{\prime}}^{2,1}} \\
\left\|\nu^{\prime}\left(\theta^{m+1}\right) \nabla \Theta^{m}\right\|_{L^{p}\left(Q_{t}\right)} \leq c\left\|\theta^{m+1}\right\|_{L^{\frac{p q}{q-p}}\left(Q_{T}\right)}\left\|\nabla \Theta^{m}\right\|_{L^{q}\left(Q_{t}\right)}
\end{gathered}
$$

and

$$
\left\|\left[\nu^{\prime}\left(\theta^{m+1}\right)-\nu^{\prime}\left(\theta^{m}\right)\right] \nabla \theta^{m}\right\|_{L^{p}\left(Q_{t}\right)} \leq c\left\|\Theta^{m}\right\|_{L^{\frac{p q}{q-p}\left(Q_{T}\right)}}\left\|\nabla \theta^{m}\right\|_{L^{q}\left(Q_{T^{\prime}}\right)} .
$$

In view of (3.15), we have

$$
\begin{aligned}
I_{3} & \leq c\left\|\Theta^{m}\right\|_{L^{\infty}\left(Q_{t}\right)}\left\|u^{m}\right\|_{\mathcal{E}_{p, T^{\prime}}^{2,1}}+c\left\|\theta^{m+1}\right\|_{L^{\frac{p q}{q-p}}\left(Q_{T}\right)}\left\|\nabla \Theta^{m}\right\|_{L^{q}\left(Q_{t}\right)} \\
& \quad+c\left\|\Theta^{m}\right\|_{L^{\frac{p q}{q-p}}\left(Q_{T}\right)}\left\|\nabla \theta^{m}\right\|_{L^{q}\left(Q_{T^{\prime}}\right)} \\
& \leq c\left\|\Theta^{m}\right\|_{L^{\infty}\left(0, t ; W^{1, q}(\Omega)\right)} \\
& \leq c\left\|U^{m-1}\right\|_{L^{p}\left(0, t ; W^{2, p}(\Omega)\right)} .
\end{aligned}
$$

Inserting (3.16)-(3.20) into (3.14), we get

$$
\begin{aligned}
\left\|U^{m}\right\|_{\mathcal{E}_{p, t}^{2,1}}+\left\|\nabla \Pi^{m}\right\|_{L^{p}\left(Q_{t}\right)} & \leq c \tilde{C}\left\|U^{m-1}\right\|_{L^{p}\left(0, t ; W^{2, p}(\Omega)\right)} \\
& \leq c \tilde{C}\left(\left\|U^{m-1}\right\|_{\mathcal{E}_{p, t}^{2,1}}+\left\|\nabla \Pi^{m-1}\right\|_{L^{p}\left(Q_{t}\right)}\right) \\
& \leq(c \tilde{C})^{m-1}\left(\left\|U^{1}\right\|_{\mathcal{E}_{p, t}^{2,1}}+\left\|\nabla \Pi^{1}\right\|_{L^{p}\left(Q_{t}\right)}\right) .
\end{aligned}
$$


We can consider $0<T^{\prime} \leq T$ such that $c \tilde{C}<1$, which implies the convergence of the series $\sum\left(\left\|U^{m}\right\|_{\mathcal{E}_{p, t}^{2,1}}+\left\|\nabla \Pi^{m}\right\|_{L^{p}\left(Q_{t}\right)}\right)$ for all $t \leq T^{\prime}$. Thus, the sequence $\left(u^{m}, \nabla \pi^{m}\right)$ converges in $\mathcal{E}_{p, T^{\prime}}^{2,1} \times L^{p}\left(Q_{T^{\prime}}\right)$. Let $u=\lim _{m \rightarrow \infty} u^{m}$ and $\nabla p=\lim _{m \rightarrow \infty} \nabla \pi^{m}$. Because $u^{m}$ converges to $u$ in $\mathcal{E}_{p, T^{\prime}}^{2,1}$, it follows from Lemma 2.2 that $\theta^{m}$ converges in $C\left(\left[0, T^{\prime}\right] ; W^{1, q}(\Omega)\right)$. Taking $\theta=\lim _{m \rightarrow \infty} \theta^{m}$, the triplet $(u, \nabla \pi, \theta)$ satisfies the system (1.2)-(1.3).

Third step. Here we show that if $A=\left(\left\|u_{0}\right\|_{W^{2-\frac{2}{p}, p}(\Omega)}+\left\|\theta_{0}\right\|_{W^{1, q}(\Omega)}\|f\|_{L^{p}\left(Q_{T}\right)}\right)$ is small enough, then $T^{\prime}=T$. To do this, recall the inequality (3.10),

$$
\Psi^{m+1}(T) \leq \tilde{C} A e^{c_{1} T^{1 / p^{\prime}} \Psi^{m}(t)}+\tilde{C} T^{\frac{1-a}{p}} \Psi^{m}(T)^{2} .
$$

Analogously to the first step, there is a constant $c>0$ such that if $\Psi^{m}(t) \leq 4 c A$, then $\tilde{C} \leq M$. Also, there exists $L_{0}>0$ such that if $A \leq L_{0}$, then

$$
4 c_{1} c T^{1 / p^{\prime}} A \leq \ln (2 c / M), 8 c A M T^{\frac{1-a}{p}} \leq 1 .
$$

In view of $(3.22)$ we have that $\Psi^{m+1}(T) \leq 4 c A$ when $\Psi^{m}(T) \leq 4 c A$. Just like the first step, it follows that $u^{m}$ and $\nabla \pi^{m}$ are uniformly bounded in $\mathcal{E}_{p, T}^{2,1}$ and $L^{p}\left(Q_{T}\right)$, respectively. Moreover, again the inequality (2.2) and the uniform boundedness of $\left\{u_{m}\right\}_{m \geq 1}$ yield

$$
\left\|\theta^{m}\right\|_{L^{\infty}\left(0, t ; W^{1, q}(\Omega)\right)} \leq\left\|\theta_{0}\right\|_{W^{1, q}(\Omega)} e^{\int_{0}^{T}\left\|\nabla u^{m}(s)\right\|_{\infty} d s} \leq c\left\|\theta_{0}\right\|_{W^{1, q}(\Omega)}, \text { for all } t \in[0, T],
$$

which implies that $\theta^{m}$ is uniformly bounded in $C\left([0, T] ; W^{1, q}(\Omega)\right)$. Proceeding as in the proof of first and second steps, we obtain the desired result.

\section{Uniqueness.}

Proof. Let $\left(u_{1}, \nabla \pi_{1}, \theta_{1}\right)$ and $\left(u_{2}, \nabla \pi_{2}, \theta_{2}\right)$ be two solutions of (1.2)-(1.3) with the same data $\theta_{0} \in W^{1, q}(\Omega), u_{0} \in W^{2-\frac{2}{p}, p}(\Omega)$, and $f \in L^{p}\left(Q_{T}\right)$. Denote $U=u_{1}-u_{2}$, $\nabla \Pi=\nabla \pi_{1}-\nabla \pi_{2}$, and $\Theta=\theta_{1}-\theta_{2}$. Then $(U, \Pi, \Theta)$ satisfies the system

$$
\left\{\begin{array}{l}
\partial_{t} U-\operatorname{div}\left(\nu\left(\theta_{1}\right)\left(\nabla U+\nabla^{*} U\right)\right)+\nabla \Pi \\
=\Theta f-U \cdot \nabla u_{2}-u_{1} \cdot \nabla U+\operatorname{div}\left(\left(\nu\left(\theta_{1}\right)-\nu\left(\theta_{2}\right)\right)\left(\nabla u_{2}+\nabla^{*} u_{2}\right)\right), \\
\operatorname{div} U=0 \\
\partial_{t} \Theta+u_{1} \cdot \nabla \Theta=-U \cdot \nabla \theta_{2} \\
U=0 \text { on } \Gamma_{T} \\
\left.(U, \Theta)\right|_{t=0}=(0,0) \text { in } \Omega .
\end{array}\right.
$$

Applying inequality (2.20), it follows that

$$
\begin{gathered}
\|U\|_{\mathcal{E}_{p, t}^{2,1}}+\|\nabla \Pi\|_{L^{p}\left(Q_{t}\right)} \leq \tilde{C}\left(\|\Theta f\|_{L^{p}\left(Q_{t}\right)}+\left\|U \cdot \nabla u_{2}\right\|_{L^{p}\left(Q_{t}\right)}+\left\|u_{1} \cdot \nabla U\right\|_{L^{p}\left(Q_{t}\right)}\right. \\
\left.\quad+\left\|\operatorname{div}\left(\nu\left(\theta_{1}\right)-\nu\left(\theta_{2}\right)\right)\left(\nabla u_{2}+\nabla^{*} u_{2}\right)\right\|_{L^{p}\left(Q_{t}\right)}\right) \\
:=\tilde{C}\left(J_{1}+J_{2}+J_{3}+J_{4}\right)
\end{gathered}
$$

for all $0 \leq t \leq T^{\prime}$, where $0<T^{\prime} \leq T$ is a common existence time. Here, in view of Lemma 2.3 , notice that $\tilde{C}$ depends continuously on $\left\|\tilde{\nu}\left(\theta_{1}\right)\right\|_{L^{\infty}\left(0, t ; W^{1, q}(\Omega)\right)}$. Let $0<\theta<1$ be such that $(1-\theta) / p=1 / 2-N / 2 p$. Now we recall the following property of the Besov spaces (see [27]):

$$
\left(\mathcal{B}_{\infty, \infty}^{1-\frac{2-N}{p}}, \mathcal{B}_{\infty, \infty}^{1-\frac{N}{p}}\right)_{\theta, 1}=\mathcal{B}_{\infty, 1}^{0} \subset L^{\infty}
$$


with

$$
\|\nabla h\|_{L^{\infty}(\Omega)} \leq c\|\nabla h\|_{\mathcal{B}_{\infty, \infty}^{1-\frac{N}{p}}}^{\theta}\|\nabla h\|_{\mathcal{B}_{\infty, \infty}^{1-\frac{2-N}{p}}}^{1-\theta} .
$$

Since $W^{2-\frac{2}{p}, p}(\Omega) \subset \mathcal{B}_{p, p}^{2-\frac{2}{p}} \subset \mathcal{B}_{\infty, \infty}^{2-\frac{2-N}{p}}$ and $W^{1, p}(\Omega) \subset \mathcal{B}_{\infty, \infty}^{1-\frac{N}{p}}$, the Hölder and Young inequalities yield

$$
\begin{aligned}
\|\nabla U\|_{L^{p}\left(0, t ; L^{\infty}(\Omega)\right)} & \leq c\left(\int_{0}^{t}\|\nabla U\|_{\mathcal{B}_{\infty, \infty}^{1-\frac{N}{p}}}^{\theta p}\|\nabla U\|_{\mathcal{B}_{\infty, \infty}^{1-\frac{2-N}{p}}}^{(1-\theta) p} d \tau\right)^{1 / p} \\
& \leq c\left(\int_{0}^{t}\|U\|_{W^{2, p}(\Omega)}^{\theta p}\|U\|_{W^{2-\frac{2}{p}}(\Omega)}^{(1-\theta) p} d \tau\right)^{1 / p} \\
& \leq c t^{\frac{1}{2}-\frac{N}{2 p}}\|U\|_{L^{p}\left(0, t ; W^{2, p}(\Omega)\right)}^{\theta}\|U\|_{L^{\infty}\left(0, t ; W^{2-\frac{2}{p}}(\Omega)\right)}^{1-\theta} \\
& \leq c t^{\frac{1}{2}-\frac{N}{2 p}}\left(\|U\|_{L^{p}\left(0, t ; W^{2, p}(\Omega)\right)}+\|U\|_{L^{\infty}\left(0, t ; W^{2-\frac{2}{p}}(\Omega)\right)}\right) .
\end{aligned}
$$

We conclude from (3.24) that

$$
\begin{aligned}
J_{3} & =\left\|u_{1} \cdot \nabla U\right\|_{L^{p}\left(Q_{t}\right)} \leq\left\|u_{1}\right\|_{L^{\infty}\left(0, t ; L^{p}(\Omega)\right)}\|\nabla U\|_{L^{p}\left(0, t ; L^{\infty}(\Omega)\right)} \\
& \leq c\left\|u_{1}\right\|_{L^{\infty}\left(0, t ; L^{p}(\Omega)\right)} t^{\frac{1}{2}-\frac{N}{2 p}}\left(\|U\|_{L^{p}\left(0, t ; W^{2, p}(\Omega)\right)}+\|U\|_{L^{\infty}\left(0, t ; W^{2-\frac{2}{p}}(\Omega)\right)}\right) \\
& \leq c\left\|u_{1}\right\|_{L^{\infty}\left(0, t ; L^{p}(\Omega)\right)} t^{\frac{1}{2}-\frac{N}{2 p}}\|U\|_{\mathcal{E}_{p, t}^{2,1}}
\end{aligned}
$$

Moreover,

$$
J_{1}=\|\Theta f\|_{L^{p}\left(Q_{t}\right)} \leq\|\Theta\|_{L^{\infty}\left(Q_{t}\right)}\|f\|_{L^{p}\left(Q_{t}\right)} \leq c\|\Theta\|_{L^{\infty}\left(0, t ; W^{1, q}(\Omega)\right)}\|f\|_{L^{p}\left(Q_{t}\right)},
$$

and

$$
\begin{aligned}
J_{2} & =\left\|U \cdot \nabla u_{2}\right\|_{L^{p}\left(Q_{t}\right)} \\
& \leq\left\|\nabla u_{2}\right\|_{L^{p}\left(0, t ; L^{\infty}(\Omega)\right)}\|U\|_{L^{\infty}\left(0, t ; L^{p}(\Omega)\right)} \\
& \leq\left\|\nabla u_{2}\right\|_{L^{p}\left(0, t ; L^{\infty}(\Omega)\right)}\|U\|_{\mathcal{E}_{p, t}^{2,1} .}
\end{aligned}
$$

In view of the equality

$$
\begin{aligned}
\operatorname{div}\left(\left(\nu\left(\theta_{1}\right)-\nu\left(\theta_{2}\right)\right)\left(\nabla u_{2}+\nabla^{*} u_{2}\right)\right)=\left(\nu\left(\theta_{1}\right)-\nu\left(\theta_{2}\right)\right) \nabla\left(\nabla u_{2}+\nabla^{*} u_{2}\right) & \\
& +\nu^{\prime}\left(\theta_{1}\right) \nabla \Theta+\left[\nu^{\prime}\left(\theta_{1}\right)-\nu^{\prime}\left(\theta_{2}\right)\right] \nabla \theta_{2},
\end{aligned}
$$

we can use the Hölder inequality and the hypotheses on $\nu$ in order to bound $J_{4}$ as follows:

$$
\begin{gathered}
J_{4} \leq c k_{1}\|\Theta\|_{L^{\infty}\left(Q_{t}\right)}\left\|u_{2}\right\|_{L^{p}\left(0, t ; W^{2, p}(\Omega)\right)}+c k_{1}\left\|\theta_{1}\right\|_{L^{p}\left(0, t ; L^{\frac{p q}{q-p}}(\Omega)\right)}\|\nabla \Theta\|_{L^{\infty}\left(0, t ; L^{q}(\Omega)\right)} \\
\quad+c k_{1}\|\Theta\|_{L^{\frac{p q}{q-p}}\left(Q_{t}\right)}\left\|\nabla \theta_{2}\right\|_{L^{q}\left(Q_{t}\right)} \\
\leq c\left(\left\|u_{2}\right\|_{L^{p}\left(0, t ; W^{2, p}(\Omega)\right)}+\left\|\theta_{1}\right\|_{L^{p}\left(0, t ; L^{\frac{p q}{q-p}}(\Omega)\right)}+\left\|\nabla \theta_{2}\right\|_{L^{q}\left(Q_{t}\right)}\right)\|\Theta\|_{L^{\infty}\left(0, t ; W^{1, q}(\Omega)\right)} .
\end{gathered}
$$

Working as in the proof of inequality (3.15), we get

$$
\|\Theta\|_{L^{\infty}\left(0, t ; W^{1, q}(\Omega)\right)} \leq c\|U\|_{\mathcal{E}_{p, t}^{2,1}}
$$


Inserting the inequalities (3.25)-(3.28) into (3.23) we obtain

$$
\begin{array}{r}
\|U\|_{\mathcal{E}_{p, t}^{2,1}}+\|\nabla \Pi\|_{L^{p}\left(Q_{t}\right)} \leq c \tilde{C}\left\{\|f\|_{L^{p}\left(Q_{t}\right)}+\left\|u_{2}\right\|_{L^{p}\left(0, t ; W^{2, p}(\Omega)\right)}+\left\|u_{1}\right\|_{L^{\infty}\left(0, t ; L^{p}(\Omega)\right)} t^{\frac{1}{2}-\frac{n}{2 p}}\right. \\
\left.+\left\|\theta_{1}\right\|_{L^{p}\left(0, t ; L^{\frac{p q}{q-p}}(\Omega)\right)}+\left\|\nabla \theta_{2}\right\|_{L^{q}\left(Q_{t}\right)}\right\}\|U\|_{\mathcal{E}_{p, t}^{2,1}}
\end{array}
$$

for all $t \in\left[0, T^{\prime}\right]$. Similar to the existence part, we have that $(c \tilde{C}) \leq M$ where $M>0$ depends on the fixed time $T^{\prime}$, but not on $t \in\left(0, T^{\prime}\right)$. Taking $t=t_{0}$ small enough in such a way that the bracketed term in (3.29) is less than $\frac{1}{2 c}$, we get

$$
\|U\|_{\mathcal{E}_{p, t}^{2,1}}+\|\nabla \Pi\|_{L^{p}\left(Q_{t}\right)} \equiv 0, \text { for all } t \in\left[0, t_{0}\right] .
$$

Therefore $\|\Theta\|_{L^{\infty}\left(0, t ; W^{1, q}(\Omega)\right)} \equiv 0$ on $\left[0, t_{0}\right]$. Now, from standard arguments, it follows that $\|U\|_{\mathcal{E}_{p, t}^{2,1}}+\|\nabla \Pi\|_{L^{p}\left(Q_{t}\right)}+\|\Theta\|_{L^{\infty}\left(0, t ; W^{1, q}(\Omega)\right)}=0$ for all $t \in\left[0, T^{\prime}\right]$.

\section{The heat conductivity vanishing}

The aim of this section is to prove Theorem 1.3.

\subsection{Proof of Proposition 1.2.}

Proof. The proof of Proposition 1.2 can be achieved through an iterative procedure. Firstly, like in Lemma 2.3, we consider the following two linear problems related to $(1.7)-(1.8)$ :

$$
\left\{\begin{aligned}
\partial_{t} u-\operatorname{div}\left(\nu(\theta)\left(\nabla u+\nabla^{*} u\right)\right)+\nabla \pi & =F \text { in } Q_{T} \\
\operatorname{div} u & =0 \text { in } Q_{T} \\
u & =0 \text { on } \Gamma_{T} \\
\left.u\right|_{t=0} & =u_{0} \text { in } \Omega
\end{aligned}\right.
$$

and

$$
\left\{\begin{aligned}
\partial_{t} \theta-\operatorname{div}(\kappa(\theta)(\nabla \theta)) & =G \text { in } \Omega \times(0, T), \\
\theta & =0 \text { on } \Gamma_{T}, \\
\left.\theta\right|_{t=0} & =\theta_{0} \text { in } \Omega .
\end{aligned}\right.
$$

Using the unique strong solution $(u, \nabla \pi) \in \mathcal{E}_{p, T}^{2,1} \times L^{p}\left(Q_{T}\right)$ for (4.1) and the solution $\theta \in \mathcal{E}_{p, T}^{2,1}$ for (4.2), we can construct a strong solution of (1.7)-(1.8) according to the following iteration scheme. Choosing $u^{0}=u_{0}$ and $\theta^{0}=\theta_{0}$, let $u^{m}, \pi^{m}, \theta^{m}$ be such that $($ for $m \in \mathbb{N}$ )

$$
\left\{\begin{aligned}
\partial_{t} u^{m+1}-\operatorname{div}\left(\nu\left(\theta^{m+1}\right)\left(\nabla u^{m+1}+\nabla^{*} u^{m+1}\right)\right)+\nabla \pi^{m+1} & =\theta^{m} f-u^{m} \cdot \nabla u^{m} \text { in } Q_{T}, \\
\operatorname{div} u^{m+1} & =0 \text { in } Q_{T} \\
\partial_{t} \theta^{m+1}-\operatorname{div}\left(\kappa\left(\theta^{m+1}\right)\left(\nabla \theta^{m+1}\right)\right) & =-u^{m} \cdot \nabla \theta^{m} \text { in } Q_{T} \\
\left(u^{m+1}, \theta^{m+1}\right) & =0 \text { on } \Gamma_{T} \\
\left.\left(u^{m+1}, \theta^{m+1}\right)\right|_{t=0} & =\left(u_{0}, \theta_{0}\right) \text { in } \Omega .
\end{aligned}\right.
$$

Then, the existence of a strong solution for (1.7)-(1.8) is obtained as the limit of the sequence $\left(u^{m}, \pi^{m}, \theta^{m}\right)$. The details are left to the reader, who is referred to [4] where an analogous result is proved for the case of incompressible micropolar fluids. 


\subsection{Proof of Theorem 1.3.}

Proof. Let $\left(u_{1}, \nabla \pi_{1}, \theta_{1}\right)$ be the local strong solution for (1.2)-(1.3) given by Theorem 1.1 and let $\left(u_{2}, \nabla \pi_{2}, \theta_{2}\right)$ be the one for (1.7)-(1.8) given by Proposition 1.2 (with $g=0$ ), both with the same data $\left(\theta_{0}, u_{0}\right) \in W^{2-\frac{2}{p}, p}(\Omega) \times W^{2-\frac{2}{p}, p}(\Omega)$ and $f \in$ $L^{p}\left(Q_{T}\right)$. Let $\left[0, T^{\prime}\right]$ be a common existence interval of $\left(u_{1}, \nabla \pi_{1}, \theta_{1}\right)$ and $\left(u_{2}, \nabla \pi_{2}, \theta_{2}\right)$. Denoting $U=u_{1}-u_{2}, \Pi=\pi_{1}-\pi_{2}$, and $\Theta=\theta_{1}-\theta_{2}$, we have that

$$
\left\{\begin{array}{l}
\partial_{t} U-\operatorname{div}\left(\nu\left(\theta_{1}\right)\left(\nabla U+\nabla^{*} U\right)\right)+\nabla \Pi \\
=\Theta f-U \cdot \nabla u_{2}-u_{1} \cdot \nabla U+\operatorname{div}\left(\left(\nu\left(\theta_{1}\right)-\nu\left(\theta_{2}\right)\right)\left(\nabla u_{2}+\nabla^{*} u_{2}\right)\right) \\
\operatorname{div} U=0 \\
\partial_{t} \Theta+u_{2} \cdot \nabla \Theta+U \cdot \nabla \theta_{1}+\operatorname{div}\left(\kappa\left(\theta_{2}\right) \nabla \theta_{2}\right)=0 \\
(U, \theta)=0 \text { on } \Gamma_{T} \\
\left.(U, \Theta)\right|_{t=0}=(0,0) \text { in } \Omega
\end{array}\right.
$$

Taking the $L^{2}$-product with $U$ in equation $(4.4)_{1}$, using $\operatorname{div}\left(u_{1}\right)=0$, and noting that

$$
-\int_{\Omega} \operatorname{div}\left(\nu\left(\theta_{1}\right)\left(\nabla U+\nabla^{*} U\right)\right) U d x \geq \beta_{0}\|\nabla U(t)\|_{L^{2}(\Omega)}^{2},
$$

we get

$$
\begin{aligned}
\frac{1}{2} \frac{d}{d t}\|U(t)\|_{L^{2}(\Omega)}^{2}+\beta_{0}\|\nabla U(t)\|_{L^{2}(\Omega)}^{2} \leq \int_{\Omega} \Theta & f U d x-\int_{\Omega}\left(U \cdot \nabla u_{2}\right) U d x \\
& +\int_{\Omega} \operatorname{div}\left(\left(\nu\left(\theta_{1}\right)-\nu\left(\theta_{2}\right)\right)\left(\nabla u_{2}+\nabla^{*} u_{2}\right)\right) U d x \\
\leq\left\|\nabla u_{2}(t)\right\|_{L^{\infty}(\Omega)}\|U(t)\|_{L^{2}(\Omega)}^{2} & \\
& +\|f(t)\|_{L^{p}(\Omega)}\|\Theta(t)\|_{L^{2}(\Omega)}\|U(t)\|_{L^{\frac{2 p}{p-2}}(\Omega)} \\
& -\int_{\Omega}\left(\left(\nu\left(\theta_{1}\right)-\nu\left(\theta_{2}\right)\right)\left(\nabla u_{2}+\nabla^{*} u_{2}\right)\right) \nabla U d x .
\end{aligned}
$$

Using $W^{1,2}(\Omega) \subset L^{\frac{2 p}{p-2}}(\Omega)$ and Young's inequality, we have

$$
\begin{aligned}
& \|f(t)\|_{L^{p}(\Omega)}\|\Theta(t)\|_{L^{2}(\Omega)}\|U(t)\|_{L^{\frac{2 p}{p-2}}(\Omega)} \\
\leq & c\|f(t)\|_{L^{p}(\Omega)}\|\Theta(t)\|_{L^{2}(\Omega)}\|U(t)\|_{W^{1,2}(\Omega)} \\
\leq & c \max \left\{1,\|f(t)\|_{L^{p}(\Omega)}^{2}\right\}\left(\|\Theta(t)\|_{L^{2}(\Omega)}^{2}+\|U(t)\|_{L^{2}(\Omega)}^{2}\right)+\frac{\beta_{0}}{4}\|\nabla U(t)\|_{L^{2}(\Omega)}^{2} .
\end{aligned}
$$

The inequality (3.19) and Hölder's inequality yield

$$
\begin{aligned}
\left|\int_{\Omega}\left(\left(\nu\left(\theta_{1}\right)-\nu\left(\theta_{2}\right)\right)\left(\nabla u_{2}+\nabla^{*} u_{2}\right)\right) \nabla U d x\right| & \leq \int_{\Omega}\left(k_{1}\left(\left|\theta_{1}\right|+\left|\theta_{2}\right|\right)|\Theta|\right)\left(\left|\nabla u_{2}+\nabla^{*} u_{2}\right|\right)|\nabla U| d x \\
& \leq c\left(\left\|\theta_{1}\right\|_{L^{\infty}\left(Q_{T^{\prime}}\right)}+\left\|\theta_{2}\right\|_{L^{\infty}\left(Q_{T^{\prime}}\right)}\right) \\
& \times\|\Theta\|_{L^{2}(\Omega)}\left\|\nabla u_{2}\right\|_{L^{\infty}(\Omega)}\|\nabla U\|_{L^{2}(\Omega)} \\
& \leq c\|\Theta\|_{L^{2}(\Omega)}\left\|\nabla u_{2}\right\|_{L^{\infty}(\Omega)}\|\nabla U\|_{L^{2}(\Omega)} .
\end{aligned}
$$

In view of (4.7) and (4.8), the inequality (4.6) gives us

$$
\frac{1}{2} \frac{d}{d t}\|U(t)\|_{L^{2}(\Omega)}^{2}+\frac{3 \beta_{0}}{4}\|\nabla U(t)\|_{L^{2}(\Omega)}^{2}
$$




$$
\begin{aligned}
& \leq c \max \left\{1,\|f(t)\|_{L^{p}(\Omega)}^{2}\right\} \times\left(\|\Theta(t)\|_{L^{2}(\Omega)}^{2}+\|U(t)\|_{L^{2}(\Omega)}^{2}\right) \\
& \quad+\left\|\nabla u_{2}\right\|_{L^{\infty}(\Omega)}\|U\|_{L^{2}(\Omega)}^{2}+c\|\Theta\|_{L^{2}(\Omega)}\|\nabla U\|_{L^{2}(\Omega)}\left\|\nabla u_{2}\right\|_{L^{\infty}(\Omega)} \\
& \leq c\left(1+\|f(t)\|_{L^{p}(\Omega)}^{2}\right)\left(\|U\|_{L^{2}(\Omega)}^{2}+\|\Theta\|_{L^{2}(\Omega)}^{2}\right)+\frac{\beta_{0}}{4}\|\nabla U(t)\|_{L^{2}(\Omega)}^{2},
\end{aligned}
$$

because $\left\|\nabla u_{2}\right\|_{L^{\infty}\left(Q_{T^{\prime}}\right)} \leq\left\|u_{2}\right\|_{\mathcal{E}_{p, T^{\prime}}^{2,1}} \leq c$. Taking now the $L^{2}$-product with $\Theta$ in $(4.4)_{3}$, and using the Young and Hölder inequalities, we obtain

$$
\begin{aligned}
& \frac{1}{2} \frac{d}{d t}\|\Theta(t)\|_{L^{2}(\Omega)}^{2}+\left\|\sqrt{\kappa\left(\theta_{2}\right)} \nabla \Theta(t)\right\|_{L^{2}(\Omega)}^{2} \\
= & -\int_{\Omega} U \cdot \nabla \theta_{1} \Theta d x+\int_{\Omega} \kappa\left(\theta_{2}\right) \nabla \theta_{1} \nabla \Theta \\
\leq & \left\|\nabla \theta_{1}\right\|_{L^{q}(\Omega)}\|U\|_{L^{\frac{2 q}{q-2}(\Omega)}}\|\Theta\|_{L^{2}(\Omega)}+\tilde{\kappa}^{1 / 2}\left\|\nabla \theta_{1}\right\|_{L^{2}(\Omega)}\left\|\sqrt{\kappa\left(\theta_{2}\right)} \nabla \Theta\right\|_{L^{2}(\Omega)} \\
\leq & c\left(\|U\|_{L^{2}(\Omega)}+\|\nabla U\|_{L^{2}(\Omega)}\right)\|\Theta\|_{L^{2}(\Omega)}+\tilde{\kappa}^{1 / 2}\left\|\nabla \theta_{1}\right\|_{L^{2}(\Omega)}\left\|\sqrt{\kappa\left(\theta_{2}\right)} \nabla \Theta\right\|_{L^{2}(\Omega)} \\
\leq & c\left(\|U\|_{L^{2}(\Omega)}^{2}+\|\Theta\|_{L^{2}(\Omega)}^{2}\right)+\frac{3 \beta_{0}}{8}\|\nabla U\|_{L^{2}(\Omega)}^{2}+\tilde{\kappa}^{1 / 2}\left\|\nabla \theta_{1}\right\|_{L^{2}(\Omega)}\left\|\sqrt{\kappa\left(\theta_{2}\right)} \nabla \Theta\right\|_{L^{2}(\Omega)} \\
\leq & c\left(\|U\|_{L^{2}(\Omega)}^{2}+\|\Theta\|_{L^{2}(\Omega)}^{2}\right)+\frac{3 \beta_{0}}{8}\|\nabla U\|_{L^{2}(\Omega)}^{2}+\frac{\tilde{\kappa}}{2}\left\|\nabla \theta_{1}\right\|_{L^{2}(\Omega)}^{2}+\frac{1}{2}\left\|\sqrt{\kappa\left(\theta_{2}\right)} \nabla \Theta\right\|_{L^{2}(\Omega)}^{2} .
\end{aligned}
$$

We deduce from (4.9)-(4.10) that

$$
\begin{aligned}
\frac{d}{d t}\left(\|U(t)\|_{L^{2}(\Omega)}^{2}+\|\Theta(t)\|_{L^{2}(\Omega)}^{2}\right) \leq c(1 & \left.+\|f(t)\|_{L^{p}(\Omega)}^{2}\right)\left(\|U(t)\|_{L^{2}(\Omega)}^{2}+\|\Theta(t)\|_{L^{2}(\Omega)}^{2}\right) \\
& +\frac{\tilde{\kappa}}{2}\left\|\nabla \theta_{1}\right\|_{L^{2}(\Omega)}^{2} .
\end{aligned}
$$

From Gronwall's inequality, the estimate (4.11) implies

$$
\begin{aligned}
\|U(t)\|_{L^{2}(\Omega)}^{2}+\|\Theta(t)\|_{L^{2}(\Omega)}^{2} & \leq\left(\|U(0)\|_{L^{2}(\Omega)}^{2}+\|\Theta(0)\|_{L^{2}(\Omega)}^{2}+\frac{\tilde{\kappa}}{2} \int_{0}^{T^{\prime}}\left\|\nabla \theta_{1}(s)\right\|_{L^{2}(\Omega)}^{2} d s\right) \\
& \times e^{\int_{0}^{t} c\left(1+\|f(s)\|_{L^{p}(\Omega)}^{2}\right) d s} \\
& \leq\left(\frac{\tilde{\kappa}}{2} \int_{0}^{T^{\prime}}\left\|\nabla \theta_{1}(s)\right\|_{L^{2}(\Omega)}^{2} d s\right) e^{c \int_{0}^{T^{\prime}}\left(1+\|f(s)\|_{L^{p}(\Omega)}^{p}\right) d s} \\
& \leq c \tilde{\kappa} .
\end{aligned}
$$

Above we have used that $\|U(0)\|_{L^{2}(\Omega)}^{2}+\|\Theta(0)\|_{L^{2}(\Omega)}^{2}=0$. Therefore, for some $\alpha \in(0,1)$, we have

$$
\begin{aligned}
\|U\|_{L^{\infty}\left(0, T^{\prime} ; W^{1, p}(\Omega)\right)} & \leq c\|U\|_{L^{\infty}\left(0, T^{\prime} ; W^{2-\frac{2}{p}, p}(\Omega)\right)}^{\alpha}\|U\|_{L^{\infty}\left(0, T^{\prime} ; L^{2}(\Omega)\right)}^{1-\alpha} \\
& \leq c \tilde{\kappa}^{(1-\alpha) / 2}\|U\|_{L^{\infty}\left(0, T^{\prime} ; W^{2-\frac{2}{p}, p}(\Omega)\right)}^{\alpha} \\
& \rightarrow 0, \text { as } \tilde{\kappa} \rightarrow 0 .
\end{aligned}
$$

Analogously, one can show that $\|\Theta\|_{L^{\infty}\left(0, T^{\prime} ; W^{1, q}(\Omega)\right)} \rightarrow 0$ as $\tilde{\kappa} \rightarrow 0$. 


\section{REFERENCES}

[1] M. de Almeida and L.C.F. Ferreira, On the well posedness and large-time behavior for Boussinesq equations in Morrey spaces, Diff. Integral Eqs., 24, 719-742, 2011.

[2] H. Abels, Nonstationary Stokes system with variable viscosity in bounded and unbounded domains, Discrete Contin. Dyn. Syst. Ser. S, 3, 141-157, 2010.

[3] H. Abidi, Sur l'unicité pour le système de Boussinesq avec diffusion non linéaire, J. Math. Pures et. Appl., 91, 80-99, 2009.

[4] J.L. Boldrini, M. Durán, and M.A. Rojas-Medar, Existence and uniqueness of strong solution for the incompressible micropolar fluid equations in domains of $\mathbb{R}^{3}$, Ann. Univ. Ferrara, 56, 37-51, 2010.

[5] L. Brandolese and M.E. Schonbek, Large time decay and growth for solutions of a viscous Boussinesq system, Trans. Amer. Math. Soc. 364, 5057-5090, 2012.

[6] J.R. Cannon and E. DiBenedetto, The initial value problem for the Boussinesq equations with data in Lp, in Approximation Methods for Navier-Stokes Problems, Lecture Notes in Math., $771,129-144,1980$.

[7] D. Chae, Global regularity for the 2D Boussinesq equations with partial viscosity terms, Adv. in Math., 203, 497-513, 2006.

[8] D. Chae and O.Y. Imanuvilov, Generic solvability of the axisymmetric 3-D Euler equations and the 2-D Boussinesq equations, J. Diff. Eqs., 156, 1-17, 1999.

[9] R. Danchin, Density-dependent incompressible fluids in bounded domains, J. Math. Fluid Mech., 8, 333-381, 2006.

[10] R. Danchin and M. Paicu, Existence and uniqueness results for the Boussinesq system with data in Lorenz spaces, Physica D., 237, 1444-1460, 2008.

[11] R. Danchin and M. Paicu, Le théorème de Leray et le théorème de Fujita-Kato pour le système de Boussinesq partiellement visqueux, Bull. Soc. Math. France, 136, 261-309, 2008.

[12] R.J. DiPerna and P.L. Lions, Ordinary differential equations, transport theory and Sobolev spaces, Invent. Math., 98, 511-547, 1989.

[13] E. Feireisl and M.E. Schonbek, On the Oberbeck-Boussinesq approximation on unbounded domains, Nonlin. Par. Diff. Equ., Abel Symposia, 7, 131-168, 2012.

[14] L.C.F. Ferreira and E.J. Villamizar-Roa, On the stability problem for the Boussinesq equations in weak- $L^{p}$ spaces, Commun. Pure Appl. Anal., 9, 667-684, 2010.

[15] L.C.F. Ferreira and E.J. Villamizar-Roa, Existence of solutions to the convection problem in a pseudomeasure-type space, Proc. R. Soc. Lond. Ser. A Math. Phys. Eng. Sci., 464, 19831999, 2008.

[16] L.C.F. Ferreira and E.J. Villamizar-Roa, Well-posedness and asymptotic behaviour for the convection problem in $\mathbb{R}^{n}$, Nonlinearity, 19, 2169-2191, 2006.

[17] A. Graham, Shear patterns in a unstable layer of air, Philos. Trans. Roy. Soc. London Ser. A, 232, 285-296, 1933.

[18] T. Hishida, On a class of stable steady flows to the exterior convection problem, J. Diff. Eqs., 141, 54-85, 1997.

[19] T. Hmidi and S. Keraani, On the global well-possedness of the two-dimensional Boussinesq system with a zero diffusivity, Adv. Diff. Eqs., 12, 461-480, 2007.

[20] T.Y. Hou and C. Li, Global well-posedness of the viscous Boussinesq equations, Discrete Cont. Dyn. Syst. A., 12, 1-12, 2005.

[21] O. Ladyszhenskaya and V.A. Solonnikov, Unique solvability of an initial and boundary value problem for viscous incompressible nonhomogeneous fluids, Zap. Nauĉn Sem.-Leningrado Otdel Math. Inst. Steklov., 52, 52-109, 1976. (English Translation, J. Soviet Math., 9, 697-749, 1978).

[22] M. Lai, R. Pan, and K. Zhao, Initial boundary value problem for 2D viscous Boussinesq equations, Arch. Rational Mech. Anal., 199, 739-760, 2011.

[23] S. Lorca and J.L. Boldrini, The initial value problem for a generalized Boussinesq model, Nonlinear Anal., 36, 457-480, 1999.

[24] A. Majda, Introduction to PDEs and Waves for the Atmosphere and Ocean, Courant Lect. Notes Math., American Mathematical Society/CIMA, 9, 2003.

[25] H. Morimoto, Nonstationary Boussinesq equations, J. Fac. Sci. Univ. Tokyo Sect. IA Math., 39, 61-75, 1992.

[26] J. Pedloski, Geophysical Fluid Dynamics, Springer-Verlag, New York, 1978.

[27] H. Triebel, Interpolation Theory, Function Spaces, Differential Operators, North Holland, 1978.

[28] D.L. Turcotte and G. Schubert, Geodynamics: Applications of Continuum Physics to Geological Problems, JohnWiley and Sons, 1982.

[29] E.J. Villamizar-Roa and E. Ortega-Torres, On a generalized Boussinesq model around a rotat- 
ing obstacle: Existence of strong solutions, Discrete Contin. Dyn. Syst. Ser. B, 15, 825-847, 2011. 